tents are removed, and it will be found that the abdominal distension gradually decreases. As a rule the patient will be found to tolerate the indwelling duodenal tube very well; nevertheless I prescribe as a routine $1 / 6$ grain morphine six-hourly.

The patient cbtains prompt relief from vomiting and from the discomfort due to abdominal distension. It is possible, and indeed preferable, to allow fluids by mouth as soon as the apparatus is functioning. It gives the patient a good deal of comfort and helps to keep the mouth fresh, and at the same time it does no harm, since the fluid is promptly removed from the stomach by the indwelling tube. This " intestinal decompression" is kept up until the abdominal distension has completely disappeared and the bowels have been opened naturally, an event which usually takes place about the fourth day after the treatment is begun.

\section{Maintaining the Circulation}

The circulation is best maintained by giving normal saline intravenously by the continuous drip method. About six to eight pints a day are usually sufficient for an adult, but the amount can be regulated by the quantity of urine passed, which should not be less than one and a half pints in twenty-four hours. The saline should be made up from trebly distilled water, in which case it will be found that the drip can be kept up without any reaction or local phlebitis for a week or more, if necessary.

Once the bowels have moved naturally and the distension has disappeared the duodenal tube can be removed and the patient may be allowed to take small drinks by mouth, beginning with one ounce of water hourly for the first twenty-four hours. This is gradually increased and at the same time the amount of saline given is decreased. Any return cf distension or vomiting is unusual, and is treated by reinserting the duodenal tube.

\section{Results of Treatment}

The immediate improvement in the patient's condition following the institution of this treatment is not spectacular. Vomiting is at once refieved and the discomfort due to the abdominal distensicn is lessened as the intestinal contents are siphoned off. The distension gradually diminishes, but the pulse rate does not fall appreciably during the first two or three days. In the majority of cases the condition is clearing up satisfactorily by the fourth day, but in other cases the period is prolonged, and two cases in my experience have gone eight and twelve days respectively before improving. It is apparent that the intestinal muscle does not recover its function rapidly, hence the importance of resting the intestinal tract as completely as possible during the recovery phase. For this reason I believe that purgatives, enzmata, " stimulant" drugs, etc., are contraindicated in the treatment of the established case of paralytic ileus.

The results of this treatment in a short series of cases have been most encouraging. In ten cases of paralytic ileus not associated with peritonitis there has been one death; this oscurred after the paralytic ileus had cleared up, and was, due to a pulmonary embolus. The opportunities of treating cases associated with peritonitis have not been so numerous: five cases have been treated with one death-this patient, a girl with general peritonitis due to acute appendicitis, died with all the signs and symptoms of an acute peritonitis. Abdominal distension, which had been marked at operation, had almost disappeared under treatment.

Many other cases, not included in the above series, have been treated by my colleagues and by the senior residents at the Manchester Royal Infirmary with equally encouraging results.

\title{
Conclusion
}

A routine is described for the treatment of paralytic ileus which is based on the physiopathology of this condition.

The results have been a great improvement on those obtained by any other method which $I$ have seen tried.

REFERENCES

${ }^{1}$ Elman, R., and Hartmann, A. F.: Surg., Gynecol. and Obstet., 1931, Ixii, 307

2 Flman, R.: Ibid., 1933, 1vi, 175.

3 Ward, R.: Journ. Amer. Med. Assoc., 1925, lxxxiv, 1114

McIver, M. A., Benedict, E. B., and Cline, J. IV., jun.: Arch. Surg., 1926, xiii, 588.

${ }_{5}^{5}$ Paine, J. R., Carison, H. A., and Wangensteen, O. H.: Journ. Amer Med Assoc, 1933,1910

6 Wangensteen, O. H., and Paine, J. R.: Ibid., 1933, ci, 1532.

\section{CHRONIC MENINGOCOCCAL SEPTICAEMIA}

BY

A. M. STEWART-WALLACE, M.B., M.R.C.P. NEDICAL FIKST ASSISTANT AND REgISTRAR, LONDON HOSPITAL

Since Gwyn in 1899 first established meningococcaemia in a case of meningitis with arthritis, and Warfield and Walker described a case of meningococcal endocarditis with septicaemia in 1903 , it has been gradually recognized that, in addition to the short initial invasion of the blood by the meningococci which probably precedes infection of the meninges in most if not all cases of meningococcal meningitis, meningococcal septicaemia may also occur in a subacute or chronic form lasting many weeks or months, in which the meninges may or may not be involved, the septicaemia being the striking clinical feature. This type is well recognized by writers about the war period, more particularly in the French literature. Sir Humphry Rolleston, in his Lumleian Lectures in 1919, said that intermittent meningococcal fever due to septicaemia might (a) last for weeks or months without any meningeal symptoms developing; (b) follow meningitis; (c) show transient meningitic symptoms; or $(d)$ might terminate in meningitis. $\mathrm{He}$ stated that a correct diagnosis was seldom made in the absence of meningeal symptoms.

In spite of these descriptions it is felt that the more chronic forms of meningococcal septicaemia with their characteristic clinical features are not sufficiently often recognized, and do not at the present time receive generally the attention they merit, seeing that no fewer than three unconnected cases, which are described here, have occurred in the London Hospital during the last two years. These follow two further cases of a similar nature in the same wards, reported by Lawson Stote in 1929.

\section{Case Reports}

CASE I

A printer aged 25 was admitted to the London Hospital on March 31st, 1934, under Dr. Hutchison, with three weeks' history of an illness characterized at the onset by headache, and followed later by fever, pain in the legs, and tender, red cutaneous nodes on his limbs.

Histary.-Three weeks previously he had awoken in the early morning with severe frontal headache, which passed off after a few hours. He vomited once, felt weak, and stayed at home. During the next two days he developed spasms of gripping pain in the right heel, which occasionally radiated up the leg and back to the nape of the neck. He tried to work during the two succeeding days, but had a constant frontal headache, felt lethargic, and commenced to have pains in his knees and ankles. Since then he had been confined to bed with fever, profuse sweats, especially at night, pains in his legs, and severe headache during the first few days, the 
last-named subsequently becoming less marked. There was no vomiting. During this time scattered, red, painful cutaneous nodes appeared on his shins and thighs; these disappeared after a few days, and were succeeded by fresh lesions. He lost weight rapidly.

On Admission.-He was a spare youth who looked ill. His skin was moist and sweating. There was moderate pyrexia. His colour was good and his tongue moist; the fauces were slightly injected. The apex beat was not displaced; there was a short, localized, soft systolic murmur at the mitral area. No abnormal physical signs were detected in the chest or abdomen. The spleen was not palpable. The fundi, mentality, and central nervous system were normal. There was no neck rigidity, and Kernig's sign was negative.

Raised erythematous patches were scattered over the legs, arms, and chest, a few being the size of a pin's head, but the majority about as large as a thumb-nail. The larger lesions were tender, and disappeared after three to five days, leaving a faint stain, which completely vanished after a few further days. Many of the nodes had a mauvish tinge, and somewhat resembled erythema nodosum in appearance.

Course.-The raised temperature continued for a further six weeks, and was accompanied by sweating, stiffness, and pain in the joints (more particularly the knees and ankles), and several fresh outbreaks of the cutaneous lesions corresponding with slight exacerbations of pyrexia. He had no further headaches. Eventually in the eleventh week of his illness the pyrexia, the rash, and all the symptoms disappeared, and on July 7 th he was discharged fit.

Laboratory Findings. - The urine was normal. Blood cultures resulted as follows: April 9th, Type I meningococcus recovered: April 18th, sterile; April 27th, Type I meningococcus recovered; May 25th, sterile. In a nasopharyngeal swab taken on May 26th Type I meningococcus was present. The cerebro-spinal fluid examined on April 15th was clear and colourless. There was no excess of white cells. The protein content was $30 \mathrm{mg}$. per $100 \mathrm{c} . \mathrm{cm}$. The Wassermann reaction was negative. Blood counts showed that the red cells varied from $3,900,000$ to $5,000,000$, haemoglobin from 80 to 90 per cent., white cells from 7,600 to 7,800 , and polymorphs between 60 and 65 per cent. The Widal test, examination of the sputum, and an $x$-ray examination of the chest proved negative.

\section{CASE II}

A motor mechanic aged 20 was admitted to the London Hospital, under Dr. Leyton, on January 24th, 1934.

History.-Four weeks previously he had a sudden onset of severe generalized headache, which lasted three days; he stayed in, bed, but had no nausea or vomiting. He got up on the fourth day and returned to work, but had to leave early on account of severe cramping pains down the backs of both legs, causing difficulty in standing up from the sitting position, and making it necessary for him to support himself with his arms while going up or down stairs. Since then he had been almost completely confined to bed. Several attempts were made to get up, but the severe pain in the legs made walking nearly impossible. No further headaches followed. He developed pyrexia, his temperature being raised on most evenings, reaching $103^{\circ}$ on occasions. Two weeks previously a scattered, non-irritating skin eruption, consisting of rose-red papules, appeared on the trunk and limbs and slightly on the face ; the lesions were raised, slightly tender, and varied from a small papule to nodes the size of a threepenny bit. These disappeared after a few days, but were succeeded by further crops. The pyrexia continued with considerable loss of weight. For the two days before admission he again complained of some stiffness at the back of the neck.

On Admission.-The patient was well built, but showed signs of considerable loss of subcutaneous tissue. Irregular pyrexia continued. There were scattered skin lesions, on the limbs and trunk and a few on the face, with the characteristics described. The tongue was clean. The fauces were slightly injected, with some enlargement of the tonsils. There were no abnormal physical signs in the heart, chest, or abdomen, and no cardiac murmurs. The spleen was not palpable. No abnormal physical signs were found in the central nervous system, except for some slight resistance to flexing the neck during the first two days. The mental condition was normal.
Course.-The pyrexia continued for four further weeks, and the temperature became normal during the fifth week, nine weeks after the onset. A few further skin lesions appeared during this time, chiefly on the legs, the individual lesions lasting four to five days, the larger ones being slightly tender on pressure. Except for the slight neck rigidity during the first two days in hospital he showed no signs of meningeal involvement. There were no headaches or vomiting. $\mathrm{He}$ constantly complained of stiffness and pain in the legs, especially the calves, and occasionally in the arms throughout the pyrexial period.

On March 5th, following four injections of meningococcal antitoxin during the previous week, he developed a severe serum reaction with pyrexia and urticaria. This subsided two weeks later, and he was discharged fit on April 10th.

Laboratory Findings.-The urine was normal and the gonococcal fixation test negative. No meningococci were found in a throat swab on March 11th. Blood cultures produced on January 30 th a Gram-negative diplococcus, which proved to be a Group II meningococcus; on February 12th they were sterile; on March 6th a Gram-negative diplococcus was recovered; and on March 13th and 25th they were sterile. The cerebro-spinal fluid on January 31st was a clear, colourless fluid, with no excess of cells; the protein content was $30 \mathrm{mg}$. per $100 \mathrm{c.cm}$. ; the Wassermann reaction was negative. On March 15th it was clear and colourless. There were four white cells per c.mm. The protein content was $30 \mathrm{mg}$. per $100 \mathrm{c} . \mathrm{cm}$., and the chloride figure was 0.67 gram per cent. as sodium chloride. In blood counts the red cells ranged from $5,100,000$ to $4,900,000$, the haemoglobin from 85 to 65 per cent., the white cells from 12,600 to 11,200 , and the polymorphs from 81 to 71 per cent.

\section{CASE III}

A labourer aged 46 was admitted into the London Hospital on July 19th, 1935, under Dr. Rowlands, for investigation of an illness which had commenced insidiously seven months previously, and was characterized by irregular pyrexia with occasional rigors, a skin eruption resembling in some respects erythema nodosum, and pains in the legs.

History.-The patient stated that his illness had started with painful red swelling of two fingers in December, 1934, one on each hand, which he attributed to minor injuries. This was closely followed by the gradual onset of general malaise, dizziness, shivering, and weakness of the legs. $\mathrm{He}$ became feverish, and was at times delirious. In January, 1935, he was admitted to the Royal Surrey County Hospital, Guildford. All investigations proved negative. Slight temporary improvement appeared to follow teeth extraction, but he relapsed three weeks later, and since then had been confined to bed, except for a few periods from one to three days, when he tried getting up. He had had an irregular temperature, which tended to be normal or subnormal in the morning and up to $100^{\circ}$ or $101^{\circ}$ in the evening, and was accompanied once or twice a week by shivering attacks. Pains in the back of the thighs and calves were his chief complaint. No localizing symptoms were found in any system, and no pain in his head or neck. There was no vomiting. In addition to his pyrexia the only objective finding was the appearance of scattered rose-red or mauve papules, ranging in size from that of a pinhead to patches somewhat like those of erythema nodosum. They were raised and tender, lasted three or four days, and then subsided leaving a brownish stain, which persisted for ten to fourteen days and finally completely disappeared. They were confined chiefly to the arms and legs, but appeared occasionally on the chest.

Previous History.- $-\mathrm{He}$ had travelled in the East during the war. He had had malaria and influenza.

On Admission.-The pyrexia continued, accompanied by occasional rigors. $\mathrm{He}$ was thin and moderately wasted, with a marked malar flush and bright eyes. No abnormal physical signs were found in his chest, abdomen, or central nervous system, nor any cardiac murmurs. The spleen was not palpable. There were no signs of meningeal involvement. The fundi were normal. Fresh outbreaks of scattered papules and erythematous nodes with the characteristics already described continued to appear, each fresh crop tending to correspond with the peaks in the temperature. 
Laboratory Findings. - The urine was normal, except for occasional traces of albumin in October. Blood counts mostly revealed a moderate leucocytosis. The red cells ranged from $5,100,000$ to $4,500,000$, and the haemoglobin from 83 to 87 per cent. On July 23rd the leucocyte count was 15,800 ; on August 27th, 13,400; on October 10th, 23,400; and on November 8th, 10,400. The polymorphs ranged from 85 to 61 per cent. On July 29th the cerebro-spinal fluid was clear and colourless, there being two white cells per c.mm. The protein content was $25 \mathrm{mg}$. per $100 \mathrm{c} . \mathrm{cm}$. The Wassermann reaction was negative, and the Lange curve was 11222100 . Blood cultures taken on July 19th and October 4th were sterile. Negative results followed the testing of the sputum, Wassermann reaction of the blood, the blood agglutination reactions to typhoid, paratyphoid, melitensis, and abortus bacilli, and the urine; an $x$-ray of the chest and pyelograms were negative. A microscopical biopsy of the skin lesion on September 23rd showed a focal area of acute inflammation in the dermis and subcutaneous tissues. There was infiltration with neutrophil leucocytes, lymphocytes, mononuclear leucocytes, and histocytes around the small vessels and sweat glands (W. Woods)

Course.-His condition continued without altering much till the end of September. He was mentally quite normal and his general health relatively good, his only complaint being the pains in the back of his legs, which were at times severe. No headaches, vomiting, or signs of meningeal involvement. After the irregular pyrexia and skin eruptions had continued for nine months without great change he suddenly developed signs of meningitis. On September 23rd he complained of severe pain in the back of the neck and occipital region, radiating to the vertex. Pain and rigidity were caused by flexing the neck, and a positive Kernig's sign appeared. His mental condition slowly changed. At night he became disorientated and muttered to himself. $\mathrm{He}$ became drowsy, but remained rational when roused, almost to the end, though his reaction time became very slow.

Cerebro-spinal Fluid.-This became slightly turbid on September 30th, with a large excess of white cells, finely granular oxyphils predominating. No organisms were seen, and it was sterile. The protein content was $200 \mathrm{mg}$. per 100 c.cm. The Wassermann reaction was negative. On October 10th the meningococcal complement-deviation test proved negative. On October 23rd the cerebro-spinal fluid was colourless with a cobweb clot, and 250 white cells per c.mm. There were red cells with a large excess of white cells ; 66 per cent. finely granular oxyphils, and 24 per cent. mononuclear cells. There were no organisms. The fluid was sterile. The protein figure was $400 \mathrm{mg}$. per $100 \mathrm{c} . \mathrm{cm}$., and the chloride figure was 0.62 per cent. On November 12th the cerebro-spinal fluid was golden yellow and turbid, with 2,700 white cells per c.mm. The protein content was $700 \mathrm{mg}$. per $100 \mathrm{c} . \mathrm{cm}$. There were 90 per cent. finely granular oxyphils, but no organisms were seen. Cultures prcduced a Gram-negative diplococcus, a meningococcus of Group II.

Both plantar responses became extensor by October 28th. The fundi, cranial nerves, and other reflexes remained normal. $\mathrm{He}$ wasted greatly; there was increasing drowsiness, and finally coma supervened during the last week. $\mathrm{He}$ died quietly on November 28th, twelve months after the commencement of his illness, and two months after the onset of meningitis.

Post-mortem Report.-There was considerable excess of slightly turbid fluid in the subarachnoid space and ventricles. Greenish muco-pus was found in patches in the cisterna magna and basalis extending up into the cortical sulci. There was some opaque thickening and injection of the meninges overlying the cisterna magna. There was great oedema and congestion of the lungs, $\cdot$ and the liver was congested. The small, flabby spleen had an oedematous congested pulp. There was slight parenchymatous degeneration of the kidneys; and an abundant bright yellow lipoid with a broad, dark brown pigment zone, and a narrow grey medulla in the suprarenals. No endocarditis was present, but there was moderate parenchymatous degeneration of the myocardium. Moderate atheroma of the aorta was noted.

These three cases have striking features in common, and closely resemble the two other cases of subacute meningococcal septicaemia without meningitis which occurred previously in the same wards under Dr. Hutchi- son, full details of which have already been published in 1929 by Lawson Stote.

The first was that of a boy aged 7 , who had a swinging pyrexia for fifteen weeks, with pains in the arms and legs, and a rash with lesions resembling erythema nodosum on the limbs. The spleen was just palpable in the thirteenth week. No cardiac murmurs were heard, and there were no headaches or signs of meningeal involvement. A blood culture produced meningococcus Type IV. The cerebro-spinal fluid was normal, and the blood count was physiological. Complete recovery ensued.

The second was that of a boy aged 15, who for twelve weeks ran an irregular pyrexia with occasional rigors. Raised, tender, erythematous nodes appeared on the chest during the first week and later on the limbs. There were pains in the joints, but no headaches or signs of meningeal involvement, and no cardiac murmurs. The spleen was not palpable. A meningococcus, Type IV, was grown from the blcod. The cerebro-spinal fluid was normal, and the blood count physiological. Complete recovery followed.

\section{Clinical Features}

The meningococcus, therefore, can cause subacute and chronic septicaemia lasting many weeks or months without any, or with only transient signs of, meningeal involvement, presenting a clinical picture with many characteristic features. All the London Hospital cases occurred sporadically and quite independently, and in no instance could exposure to infection be established. Children or adults may be affected, and while all our cases happened to be males examples of the condition in females can be found in the literature.

The onset is usually insidious, with malaise, pain in the limbs, and pyrexia, or the illness may commence with meningitic signs. In two of the cases there was severe headache and pain in the back of the neck at the commencement, although in both instances, until the diagnosis was made by finding meningococci in the blood stream, the significance of the symptom was not appreciated, and the headache did not appear a more prominent feature than the malaise, pain in the limbs, and the fever. There is an irregular pyrexia, the temperature tending to be normal or subnormal in the morning, with evening rises up to $100^{\circ}$ to $103^{\circ}$, with occasional periods of a few days without much elevation.

Shivering attacks occurred once, or sometimes twice, a week in two cases, but there was no great accompanying rise in temperature such as is usually associated with a typical rigor. In all the cases the pulse rate seldom rose above 100, even when the temperature was high, and more frequently than not was below 90 , in this respect resembling typhoid fever. The general condition is remarkably good, although there is considerable loss of weight. The appetite is usually fair.

The skin rashes in our cases, and in the majority of reported cases, were similar in many respects ; they form a very characteristic feature of the condition. They are most frequently seen on the legs and arms, less often on the chest and face, and rarely on the abdomen and back. The lesions consist of rose-red or mauve papules and raised erythematous patches, varying in size from a pinhead to a thumb-nail, the larger ones being tender on pressure, and resembling in some respects erythema nodosum. They disappear after three to seven days, in some of the cases leaving a brown stain, which gradually fades during the next few days, and are followed by further lesions; in some instances the fresh outbreaks seem to correspond with the peaks in the temperature. Biopsy of a lesion in Case III showed no specific microscopical changes.

Pains in the legs, especially the back of the thighs and calves, and also in the knees and ankles, are a marked feature, while occasional pain may also occur in the arms. 
No redness, swelling, or tenderness is observable in the painful joints. The spleen may just be palpable, but more often is not. Meningococcal endocarditis has been described, and may complicate the septicaemia, but more frequently the valves are not affected. A necropsy on Case III after remittent pyrexia for twelve months revealed no endocarditis. Cases with metastases in joints, pleura, and conjunctivae have also been described.

\section{Occurrence of Meningitis}

Meningococcaemia may persist for many weeks or months after an attack of meningitis, but in the cases to which we more especially wish to call attention there is (i) an absence of all signs of meningeal involvement, (ii) transient signs of meningeal irritation at the onset, shown by headache, pain in the back of the neck, and perhaps some neck rigidity, the cerebro-spinal fluid in these cases remaining unchanged, or (iii) a terminal onset of meningitis, as happened in Case III after nine months' illness.

The blood count may show a leucocytosis of 12,000 to 25,000 white cells, or may be normal. There is never a leucopenia. The chronic forms of meningococcaemia are not peculiar to any group of meningococci, Types I, II, and IV being found in the present series. The organism can sometimes only be isolated from the blood with difficulty, or not at all, and a negative blood culture does not exclude the condition.

\section{Duration and Prognosis}

The pyrexia may last for many weeks or months. In our cases the pyrexial period extended over nine, eleven, twelve, fifteen, and fifty weeks respectively. Several cases continuing for five to nine months are recorded in the literature, but no reference has been found to an instance lasting for twelve months, as did Case III.

The prognosis is good. Four out of the present five cases and over 75 per cent. of those previously published have completely recovered after many weeks' or months' pyrexia. Endocarditis or the late development of meningitis, usually both, occur in almost all fatal cases. Definite endocarditis probably renders the prognosis hopeless.

\section{Conclusions}

Subacute and chronic forms of meningococcal septicaemia may last for a few weeks, or for longer periods up to nine or twelve months, signs of meningeal involvement being absent, or only transient at the onset. They are characterized by irregular pyrexia, with occasional rigors but with little elevation of the pulse rate, leucocytosis, pains in the limbs, and peculiar skin eruptions. Over 75 per cent. of these cases recover completely, but endocarditis or terminal meningitis may be a fatal complication.

The condition is seldom-diagnosed in the absence of meningeal symptoms. The organism is sometimes difficult or impossible to isolate, and negative blood cultures and a negative complement-deviation test are not necessarily conclusive. This was shown by Case III, in which repeated blood cultures were negative during the whole period of twelve months of pyrexia and rigors, the meningococcus only being recovered from the cerebrospinal fluid at the terminal stage after meningitis had eventually set in, and only thus was the diagnosis established.

It is thought that this condition probably occurs more frequently than is generally recognized, and that some cases pass undiagnosed. The skin lesions may so closely resemble erythema nodosum that perhaps some cases at present diagnosed as erythema nodosum may really be examples of meningococcal septicaemia. More particularly is this so in those cases in which the pyrexia persists for several weeks, with recurrent crops of the nodes, especi- ally if the lesions are also present on the arms, chest, or face. In a case of long-continued and obscure pyrexia a meningococcal origin should be considered as a possibility. Inquiries should be made for any of the characteristic symptoms and signs, and the appropriate laboratory investigations should be undertaken, bearing in mind that a negative blood culture is not necessarily conclusive.

I wish to express my thanks to Drs. Hutchison, Leyton, and Rowlands for their kind permission to publish their cases, and to acknowledge with gratitude Dr. Lawson Stote's previous publication of the two earlier cases from the London Hospital, the case records of which I have also referred to in the discussion of the clinical picture.

\section{BIBLIOGRAPHY}

Gwyn: Bull. Johns Hopkins Hosp., 1899, x, 112.

Rolleston: Lancet, 1919, i, 541.

Stote: Ibid., 1929, i, 701.

Warfield and Walker: Bull. Amer. Clin. Lab., Philadelphia, 1903, i, 81.

\section{VIRUSES IN THE AETIOLOGY OF SKIN DISEASES *}

BY

R. T. BRAIN, M.D., F.R.C.P.

WALTER DIXON RESEARCH SCHOLAR OF THE BRITISH MEDICAL ASSOCIATION, 1935

(From the Freedom Research Laboratory, London Hospital)

Lesions of the skin produced by filterable viruses present a variety of types. There are such lesions as warts and molluscum contagiosum in which cellular proliferation and tumour-like formation are the dominant features, anything in the nature of an inflammatory reaction being absent. There is, again, the type of lesion presented by herpes, zoster, and varicella, characterized by swelling and ballooning of the affected epidermal cells with considerable leucocytic infiltration of the epidermis, and the presence of a serous exudate, which dissociates the epidermal cells and collects beneath the stratum corneum in the form of a vesicle. In some cases, as in the pock diseases, the leucocytic infiltration reaches such dimensions that the vesicle fluid becomes frankly purulent and the fully developed lesion is a pustule.

In addition to these two main types of lesion-the hypertrophic tumour-like lesion and the vesicular or pustular-there are others. The exanthem of measles is essentially an urticarial papule in which vesicle formation is so little in evidence as to be only detectable by means of the microscope; the erythematous macule of typhus constitutes yet another type. Thus there is nothing either in the naked-eye appearance or the gross histology which can be said to be a common feature of virus lesions of the skin; further than this, similar types of lesion are produced by agents other than viruses.

\section{Pathological Manifestations}

This does not mean that histological study of a lesion is of no value, for the affected cells may present changes which are peculiar to virus lesions. These are the inclusion bodies, homogeneous or granular acidophilic masses of varying size situated either in the cytoplasm or in tho nucleus. Although inclusion bodies have not been demonstrated in the case of all viruses, available evidence suggests that they are not produced by any other type of infective agent. The nuclear inclusions produced by the viruses of herpes, zoster, and varicella, and the cytoplasmic inclusions seen in molluscum contagiosum, are good examples of this type of change.

In addition to this it may be possible to demonstrato the virus itself in smears made with material from the

* Read at the Ninth International Congress of Dermatology and Syphilis, Budapest, September, 1935. 